\title{
Extrinsic Optical Fiber Sensor for Medical Audiometric Applications
}

\author{
K. Prokopczuk*, T. Poczęsny, P. Sobotka and A.W. Domański \\ Faculty of Physics, Warsaw University of Technology, Koszykowa 75, 00-662 Warszawa, Poland
}

\begin{abstract}
The paper presents optical fiber sensor designed for medical audiometric measurements. Currently, highly precise audiometric examinations require attachment of reflective element inside the ear. We propose alternative contactless optical and non-invasive method for audiometric applications. Presented sensor utilizes extrinsic intensity modulation combined with interferometric method to measure low amplitude vibrations with flat frequency response curve and no resonance effects. Experiment was performed with tympanic membrane model stimulated by external loudspeaker. Experimental results show usage possibility of presented sensor for laryngological and audiometric tests of ear membrane response, which is important for both diagnosis and implants sensitivity fitting for people with hearing deficits.
\end{abstract}

PACS: 42.81.Pa, 42.79.Pw

\section{Introduction}

Typical audiometric examination of tympanic membrane and ossicular mobility is based on stimulating them by external sound with fixed loudness and measuring their deflection amplitude. It gives an information about flexibility of membrane, efficiency of pressure transfer between mechanisms inside ear, and finally about sensitivity of hearing. For patients with relatively good hearing membrane flexibility is high and pressure transfer is efficient. When amplitude of membrane deflection is lower than normal, it is sign of limited and impaired ability to hear. Correct adaptation of hearing aids requires information of tympanic membranes mobility.

Most highly precise audiometric and tympanometric examinations need sophisticated and complex measurement methods [1-3]. In this area, optical methods of vibration monitoring are recently becoming more popular and effective due to contactless measurement possibility [4-7]. The aim of work was to propose an optical fiber method of measuring vibration amplitude of tympanic membrane or ear ossicles stimulated by external sound. The paper presents two concepts of optical fiber sensor designed for medical audiometric measurements. Presented sensors utilize optical fibers to illuminate part of tympanic membrane surface and to collect reflected light containing information about membrane movement. Presented sensors utilize extrinsic intensity modulation (EIM) and EIM combined with interferometric method (EIMI) to measure very low amplitude vibrations with flat frequency response curve and no resonance effects.

* corresponding author; e-mail: prokopczuk@if.pw.edu.pl
To measure tympanic membrane mobility a very sensitive and accurate optical measurement method is needed. Our recent optical vibration sensing solutions utilizing EIM were focused mainly on industrial applications [8-10]. For medical applications, we decided to use EIM method due to its relatively high sensitivity and ease in illuminating small area of tympanic membrane surface.

The main issue in EIM applications is the need for calibration every time when sensor changes its operation position e.g. due to patient movement. Also during experiment we combined EIM with interferometry to minimize influence of human body natural movements on measurement results. The combination of these two methods allows for achieving a signal which contains information about amplitude in both envelope of signal and the number of fringes modulating the signal. We finally proposed the EIMI method, where sensor can be completely auto-calibrated by very simple and fast software algorithms. This method is based on extrinsic modulation of intensity and phase. This gives a possibility of using the EIMI sensor handheld and without need for patient immobilization.

\section{Principle of operation of EIM and EIMI sensors}

We present two concepts of an optoelectronic vibration sensor for audiometric applications - first one is based on typical EIM which utilizes two fibers in sensing head: first one as transmitting fiber, second one as receiving fiber. Simplified scheme of EIM sensing head was shown in Fig. 1a. This type of head works as displacement sensor - response characteristic is shown in Figs. 2 and 3. Examples of industrial applications of such sensing head were presented in previous works [8-10]. 


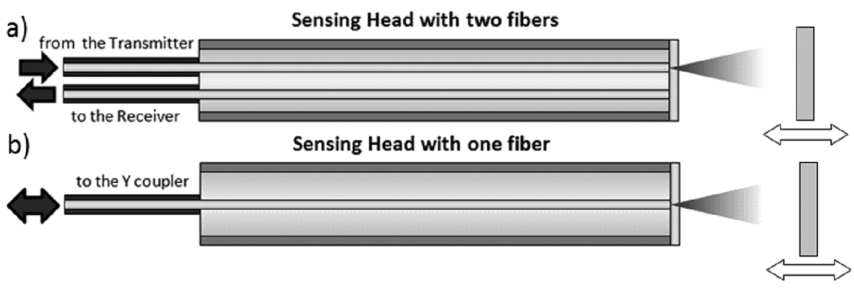

Fig. 1. Schemes of sensing heads (a) with two fibers typical EIM, (b) with one fiber - EIM combined with interferometric method (EIMI).

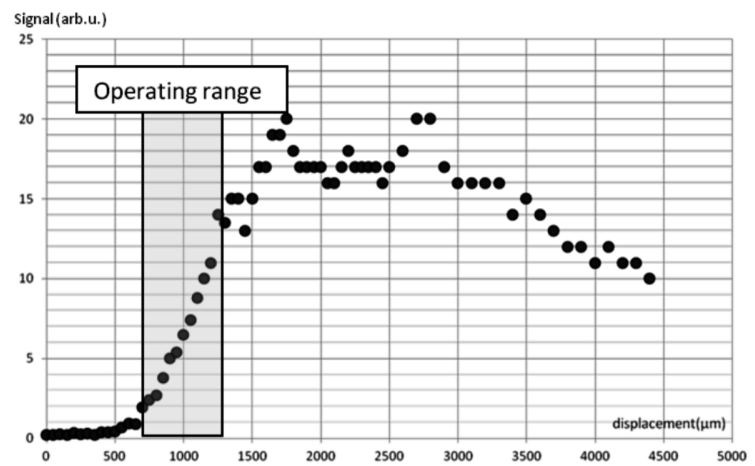

Fig. 2. Response characteristic of two-fiber head. Rising slope $(500-1500 \mu \mathrm{m})$ is linear range of possible vibration measurement. Signal irregularity in distance of $1500-3000 \mu \mathrm{m}$ is caused by surface roughness.

The second sensing head model use one fiber connected with the $Y$ coupler (Fig. $1 \mathrm{~b}$ and Fig. 4). This one fiber works as both optical signal transmitter and receiver at once. This sensing head utilizes both EIM and interferometric method.

Standard EIM method is based on measurement of the power of light returning after reflection from a tested object. The response curve consists of two slopes which can be approximated as two linear measurement ranges (Fig. 2). Rising slope gives a high sensitivity with a small

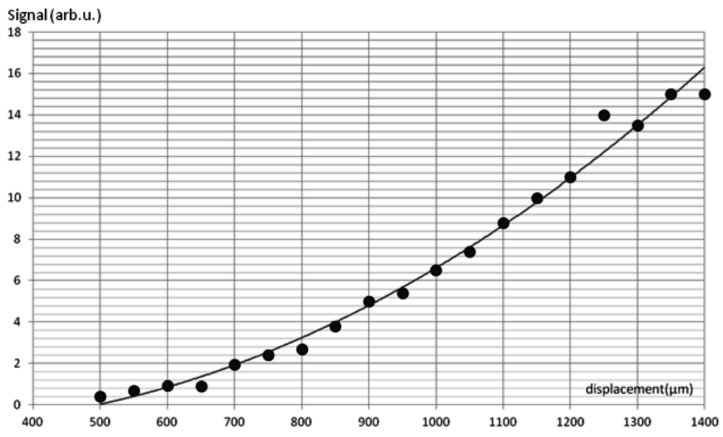

Fig. 3. Rising slope (called "near side") of response characteristic of two-fiber head. Only this slope was used in our audiometric tests due to sufficient linearity and signal repeatability in case of rough test surface.

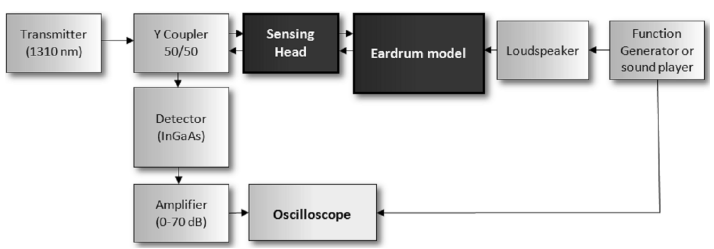

Fig. 4. Block diagram of experimental setup. Please note that in some measurements $Y$ coupler and eardrum model were removed.

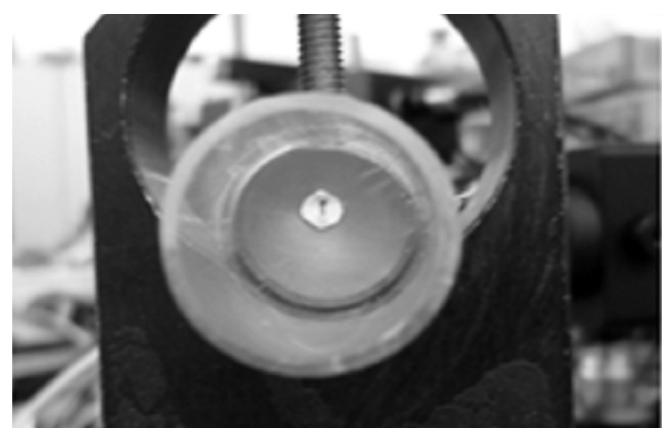

Fig. 5. Physical model of tympanic membrane.

amplitude range, falling slope gives lower sensitivity, but with larger amplitude range. We decided to use only rising slope in our EIM sensor audiometric tests.

In EIMI method there is only one slope of response curve. If the tested object moves away from the sensing head, the optical signal decreases. Additionally, the signal is modulated by interferometric pattern. The resultant signal is the product of both modulations

$$
S_{\mathrm{s}}=P_{0} \times M(x) \times I(x),
$$

where $S_{\mathrm{s}}$ is the optical signal from sensing head, $\mathrm{P}_{0}$ is the signal level at operating point, $M(x)$ is an EIM sensing factor dependent on EIM parameters, and $I(x)$ is an interferometric sensing factor dependent on wavelength of light.

Experimental setup was shown in a diagram in Fig. 4. We used a laser diode with central wavelength of $1310 \mathrm{~nm}$ as a light source. "Eardrum model" in this diagram means an ear tympanic membrane model based on flexible membrane applied to the plastic tube Fig. 5). The tympanic membrane model was made by specialist form Medical University of Warsaw. Physical model of membrane was partially transparent similarly to real tympanic membrane. InGaAs near infrared detector was used to measure output optical power. Detector was equipped with low-noise preamplifier. As an electric signal collector 10 GS/s oscilloscope was used.

Directional $Y$ coupler was used in way as it is shown in Fig. 4. Input arm was connected to sensing head, output arms were connected to laser and detector, respectively.

We used functional generator or sound player as a device controlling sound source. Reference tests were performed where speaker was used as tested (illuminated) 
objects. The other experiments were performed with speaker stimulating tympanic membrane model from distance, and optical signal was collected from a membrane surface (Fig. 6).
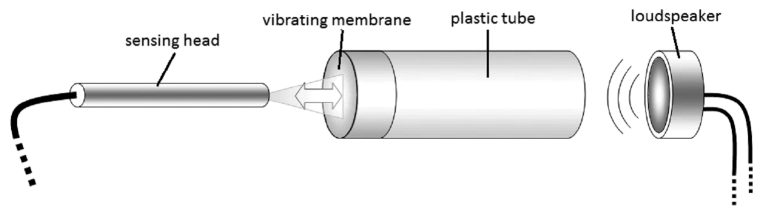

Fig. 6. Scheme of experiment, where tympanic membrane model was stimulated to vibration by loudspeaker from a distance. This situation is similar to audiometric test of tympanic membrane and ossicles mobility.

\section{Sensing head with two fibers for EIM}

In initial tests we were using a typical two-fiber EIM head as a vibration sensor. Response characteristic of EIM sensor is shown in Fig. 2. The characteristic was obtained by step shifting object away from the head. Shape of response curve is dependent on type of fibers and distance between them [8]. Tested EIM sensing head consisted of two single-mode (SM) telecommunication fibers. Use of SM instead of MM fibers allowed us to limit the impact of varied distribution of light on rough surfaces and to narrow the area of illumination.

The measurement results were obtained with operating point set on rising slope of response curve. Zoom of rising slope is shown in Fig. 3. This slope is approximately linear, but an accounting of sensitivity diversity is needed to calculate vibration amplitude precisely. It can be a significant problem in real medical applications, where human body is in constant motion.

In initial tests the sensing head was directed to the loudspeaker surface. Signal mapping was highly accurate and repeatable. Reflected light intensity was high enough to ensure also high signal to noise ratio, which is very important and sometimes hard to achieve in EIM sensing method. The comparison of electrical signal controlling loudspeaker and signal achieved from the EIM sensor was shown in Fig. 7. In Fig. 8 result for tympanic membrane model used as tested object is shown.

The main measurement problems were semi-transparence of membrane and its sensitivity to any strong air movements. However, we were able to achieve valuable signal containing information about vibration characteristics and its amplitude. By differentiating the signal momentary velocity can be calculated, and with next differentiation momentary acceleration can be calculated. To properly calculate vibration amplitude we needed to recalibrate the sensor due to change of tested surface reflectivity. Reflectance of membrane used in experiment was much lower than reflectance of loudspeaker surface. This occurred in lower optical signal power and increased noise level.

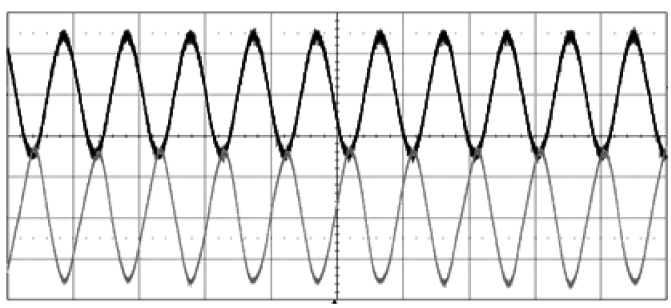

Fig. 7. Measurement with using loudspeaker as tested object. The upper curve shows signal from function generator used to control loudspeaker, the lower curve is the EIM sensor response.

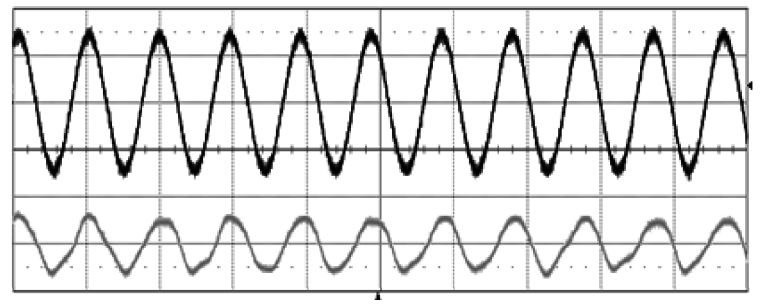

Fig. 8. Measurement with using eardrum model as tested object. The upper curve shows signal from function generator used to control loudspeaker, the lower curve is our sensor response.

Still, we were able to calculate amplitude of vibration after calibrating the sensor.

Vibration amplitude can be calculated from signal voltage level. However, this requires calibration every time when tested object is being changed or sensor is being displaced. By experimenting we have found the great advantage of combining interference phenomenon with EIM to construct EIMI sensor that will not need to be recalibrated for every patient. Additionally, EIMI sensor can be insensitive to body natural movements.

\section{Sensing head with one fiber for EIMI}

In next stage of research we focused on optimization of EIM method, so it could be utilized in real audiometric examinations. EIM method was giving good results, but it needed calibration of sensor and immobilization of sensing head and tested object. This method needed to be improved to be a practical solution for real patient examination.

In optimization stage two fibers from sensing head have been replaced by a single fiber. This one fiber was connected to directional $Y$-coupler as it is shown in Fig. 4. Due to use of coherent laser source, interference effect between light reflected from a fiber face and light reflected from a tested object appeared significantly.

We decided to utilize $I(x)$ - interferometric sensing factor dependent on wavelength of light - as a function which codes amplitude of vibration in different way than EIM factor. That gives a possibility of double sensing of amplitude for periodic signals stimulating loudspeaker 
and such sensor does not need to be calibrated every time when sensing head is being moved.

The EIMI sensor was firstly tested for displacement measurement. In this case the tested object was moved by nanometric stage. Displacement measurement was shown in Fig. 9. Signal presented in Fig. 9 shows interferometric fringes sliding during movement of the tested object by $20 \mu \mathrm{m}$. By simple calculation displacement can be designated from amount of fringes.

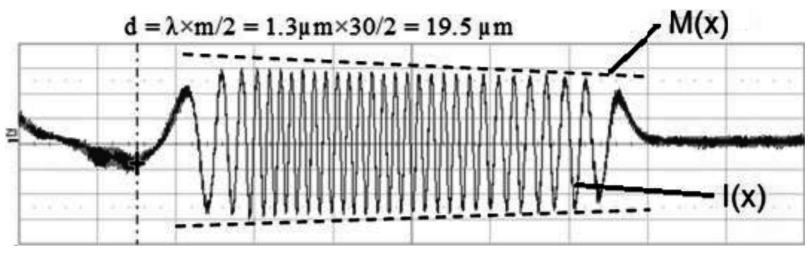

Fig. 9. Registered signal and its zoom while moving a tested object by $20 \mu \mathrm{m}$. $\lambda$ is wavelength of light and $m$ is a number of signal peaks per shift. Factors $M(x)$ and $I(x)$ occur in formula $(1)$.

Envelope of signal is achieved from EIM factor $M(x)$. Additional modulation is achieved from EIMI factor $I(x)$. Vibration measurement is shown in Fig. 10. Both factors $M(x)$ and $I(x)$ provide information about displacement or vibration amplitude. EIM factor is very easy to encode (no software is needed), but it needs a calibration every time when working distance between sensing head and tested object is being changed. With two sensing factors containing information about vibration amplitude only simple software algorithms are needed to auto-calibrate sensor.

In Fig. 11 examples of signals for increasing vibration amplitude are shown. Tones stimulating tympanic membrane model was generated by function generator. Both signals showed in Fig. 11 are achieved for sounds with the same frequency, but different amplitude. We were able to designate vibration amplitude of tympanic membrane model by counting amount of interferometric fringes in vibration period.

In case of harmonic stimulation (for example simple tones in audiometry) number of fringes can be easily calculated by thresholding algorithm or fast Fourier transform (FFT). For known and fixed stimulating sound frequency number of fringes per vibration period can be estimated. Figure 12 shows example of using the Fourier transform to estimate average number of fringes in vibration period for different amplitudes of vibration. Cutoff frequency of the Fourier spectrum is related to amplitude of vibration.

Due to small area of illumination, mapping of whole membrane surface is possible. Amplitude of vibration for whole surface can be mapped with transversal resolution dependent on fiber core diameter and e.g. additional op-

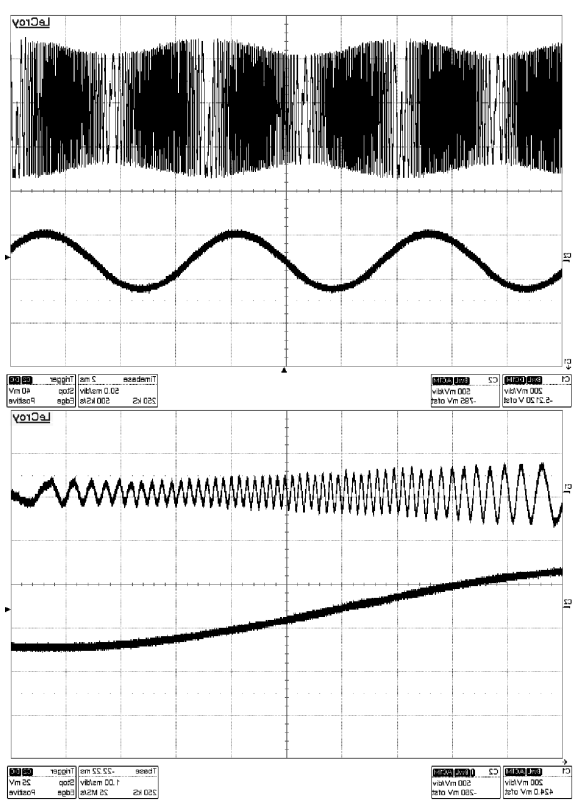

Fig. 10. Vibration measurement: lower curve - stimulating signal, upper curve - signal from EIMI sensor.

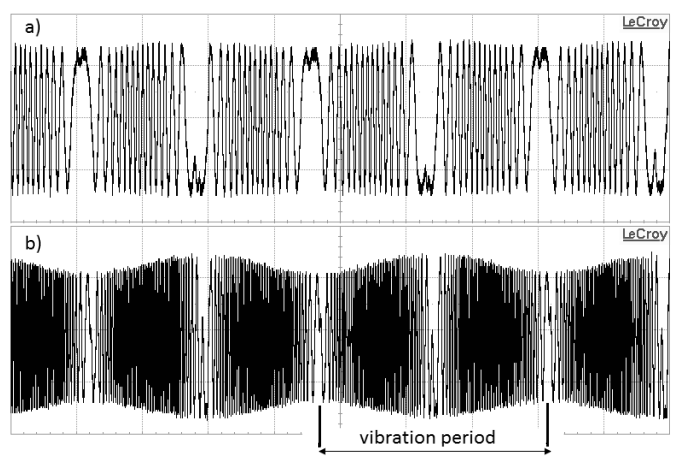

Fig. 11. Registered signals for increasing vibration amplitude: (a) function generator peak-to-peak amplitude equal to $200 \mathrm{mV}$; (b) function generator peak-to-peak amplitude equal to $600 \mathrm{mV}$; amplitude of membrane vibration can be calculated from number of fringes.

tical fiber lenses [8]. Longitudinal resolution of vibration measurement is dependent on wavelength of light.

\section{Conclusions}

Both sensing heads are able to measure: displacement, vibration characteristics, vibration amplitude and vibration frequency. Experimental results show possibility of using the EIMI sensor in laryngological tests of ear membrane response and hearing aids sensitivity fitting after further consultation and development. This method is cost-effective and creates a possibility of minimizing influence of human body natural movements on measurement results due to double amplitude coding in signal. Additionally, sensors can be used to mapping tympanic membrane surface and vibration amplitude distribution. 


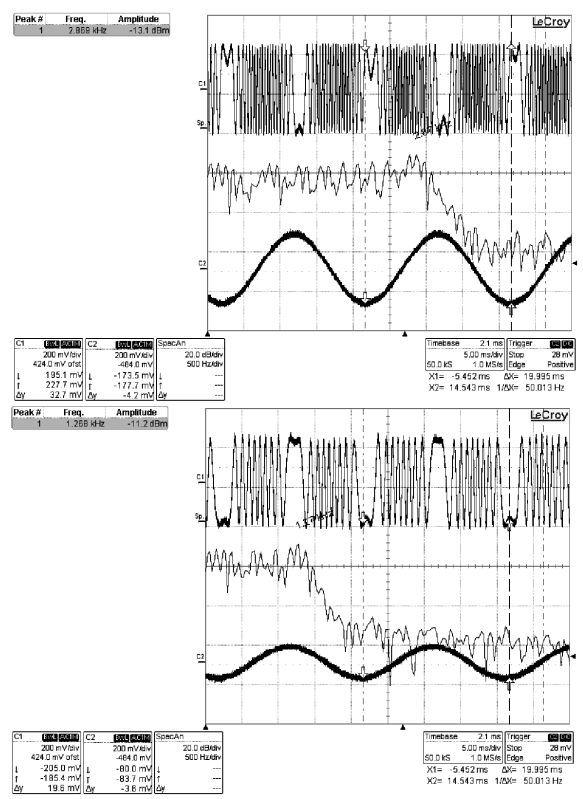

Fig. 12. Examples of Fourier transform of achieved signals for different amplitudes of vibration; vibration frequency $-50 \mathrm{~Hz}$; FFT cutoff frequencies: $1.268 \mathrm{kHz}$ and $2.869 \mathrm{kHz}$
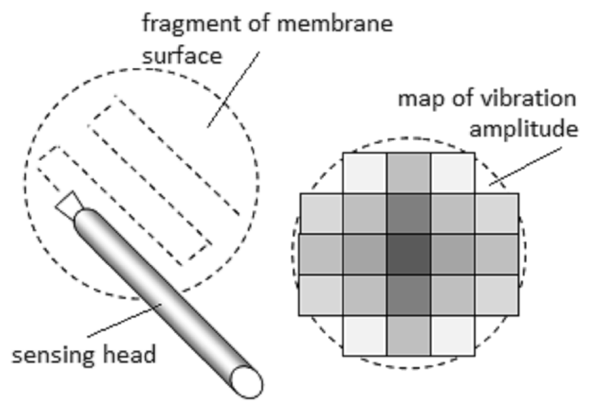

Fig. 13. Diagram of the membrane surface mapping and simplified example of vibration amplitude distribution.

\section{Acknowledgments}

This work was partially supported by The Polish National Center for Research and Development (NCBiR) under the grant N R01 0026 06/2009 and the European Social Fund implemented under the Human Capital Operational Programme (POKL), project: "Preparation and Realization of Medical Physics Specialty". The work was performed in consultation with Piotr Arcimowicz from the Medical University of Warsaw.

\section{References}

[1] N. Hato, H. Kohno, M. Okada, N. Hakuba, K. Gyo, T. Iwakura, M. Tateno, Otol. Neurotol. 27, 592 (2006).

[2] K.R. Whittemore Jr., S.N. Merchant, B.B. Poon, J.J. Rosowski, Hearing Res. 187, 85 (2004).

[3] A. Huber, T. Linder, M. Ferrazzini, S. Schmid, N. Dillier, S. Stoeckli, U. Fisch, Ann. Otol., Rhinol. Laryngol. 110, 31 (2001).

[4] Y.R. García, J.M. Corres, J. Goicoechea, J. Sensors 2010, 936487 (2010).

[5] B. Culshaw, J. Dakin, IEEE Trans. Instrument. Measur. 47, 742 (1998).

[6] K. Gut, J. Phys. IV: JP (France) 129, 109 (2005).

[7] J. Dakin, B. Culshaw, Optical Fiber Sensors - Application, Analysis, and Future Trends, Vol. 4, Artech House, Boston 1997.

[8] K. Prokopczuk, P. Lesiak, T. Poczesny, K. Rozwadowski, T.R. Wolinski, A.W. Domanski, Proc. SPIE 8082, 80823K (2011).

[9] T.R. Wolinski, D. Budaszewski, A.W. Domanski, S. Ertman, G. Goleniewski, M. Wydmanski, Proc. SPIE 6616, 66161U (2007).

[10] T.R. Wolinski, A.W. Domanski, D. Budaszewski, S. Ertman, Proc. SPIE 6585, 65850H (2007). 